\title{
Lidil
}

Revue de linguistique et de didactique des langues

$64 \mid 2021$

Le passif dans la langue parlée

\section{Salkie Raphael, Simply Chomsky}

Pressbooks.com : Simply Charly [Great Lives], New York, 2020, 154 p.

$<w w w . s i m p l y c h a r l y . c o m / w p-c o n t e n t / u p l o a d s / 2020 / 08 / S i m p l y-$

Chomsky-1597160541.pdf>.

\section{Marinette Matthey}

\section{OpenEdition}

\section{Journals}

Édition électronique

URL : https://journals.openedition.org/lidil/9433

DOI : $10.4000 /$ lidil.9433

ISSN : $1960-6052$

\section{Éditeur}

UGA Éditions/Université Grenoble Alpes

\section{Édition imprimée}

ISBN : 978-2-37747-315-1

ISSN : 1146-6480

\section{Référence électronique}

Marinette Matthey, «Salkie Raphael, Simply Chomsky », Lidil [En ligne], 64 | 2021, mis en ligne le 01 novembre 2021, consulté le 25 novembre 2021. URL : http://journals.openedition.org/lidil/9433 ; DOI : https://doi.org/10.4000/lidil.9433

Ce document a été généré automatiquement le 25 novembre 2021.

(C) Lidil 


\section{Salkie Raphael, Simply Chomsky}

Pressbooks.com : Simply Charly [Great Lives], New York, 2020, 154 p. $<$ www.simplycharly.com/wp-content/uploads/2020/08/SimplyChomsky-1597160541.pdf>.

\section{Marinette Matthey}

\section{RÉFÉRENCE}

Salkie Raphael, Simply Chomsky, Pressbooks.com : Simply Charly [Great Lives], New York, 2020, 154 p. <www.simplycharly.com/wp-content/uploads/2020/08/SimplyChomsky-1597160541.pdf>.

1 Simply Charly est une entreprise privée d'éducation en ligne, qui propose des biographies de personnalités ayant marqué leur temps (sciences et techniques, littérature, penseurs (surtout) et (quelques) penseuses célèbres...).

2 Simply Chomsky est écrit par Raphael Salkie, professeur émérite de linguistique de l'Université de Brighton, qui lui a déjà consacré plusieurs livres.

3 Comme Chomsky est davantage connu aujourd'hui pour ses prises de position politiques que pour ses modèles linguistiques, on ne s'étonnera guère de ne trouver que 5 chapitres sur 17 concernant sa vision du langage.

4 Le livre s'ouvre sur une brève biographie de Chomsky, puis sur une série de 9 mythes qui auréolent ou diabolisent Chomsky et son œuvre, où Salkie rétablit en quelques lignes la (sa) vérité. Les deux derniers mythes intéressent les sciences du langage. Le $8^{\mathrm{e}}$ est énoncé comme suit : "Chomsky is very important because he "revolutionized" the study of language.» La réalité est que Chomsky est beaucoup cité mais peu compris et que la linguistique chomskienne est en fait très minoritaire. Chomsky lui-même considère qu'il n'a rien à dire sur la pragmatique, l'enseignement des langues et encore moins sur les usages sociaux du langage, ni même sur l'évolution des langues et du langage. Or, ce sont ces domaines qui drainent la majorité des recherches aujourd'hui. Le $9^{\mathrm{e}}$ mythe concerne les lexies encore accolées par réflexe au nom de Chomsky, alors que lui-même ne les utilise plus depuis de nombreuses années: grammaire générative 
transformationnelle (GGT) ; structure profonde vs de surface, dispositif d'acquisition linguistique (LAD), locuteur-auditeur idéal, Théorie X-bar... Toutes ces notions appartiennent à des versions périmées du modèle de la grammaire générative et on n'en trouvera aucune trace dans ce livre.

L'auteur revient ensuite sur l'éducation de Chomsky (chapitre 2). Il a fréquenté enfant une école alternative fondée sur les principes de John Dewey - learning by doing et ses conceptions de l'enseignement reposent sur la coopération horizontale et non sur le bourrage de crâne vertical. Sa posture critique peut être liée à cette méfiance envers l'éducation formelle "ordinaire", qui produirait surtout des individus sachant bien parler, et qui se considèrent dès lors comme supérieurs alors qu'ils sont surtout endoctrinés, voire stupides.

6 Les convictions libertaires de Chomsky (objet du $3^{\mathrm{e}}$ chapitre) sont aussi à mettre en relation avec l'héritage de Dewey. Tout pouvoir doit pouvoir justifier son utilité, et s'il ne le peut pas, il faut le supprimer. Les structures de pouvoir hiérarchisées au sein des organisations publiques ou privées sont souvent taxées par Chomsky de fascistes (ou de «tyrannies privées ») dans le sens où chaque agent est aveugle à tout ce qui n'est pas immédiatement au-dessus de lui (obéissance à son supérieur) ou en dessous de lui (exercice du pouvoir sur le subordonné).

7 Le chapitre 4 aborde la question de la propagande, ou de la fabrication du consentement au sein des démocraties, via la proximité des médias et des élites économiques qui les financent par la publicité (ou qui les achètent).

8 Le titre du chapitre 10 est une question « What is language? ». La réponse est donnée en exergue par une citation de 2016 de Chomsky que je traduis et reformule : la propriété fondamentale du langage humain est un arrangement de constituants linguistiques hiérarchisés au sein d'une structure non bornée, arrangement qui fonctionne grâce à deux interfaces: une tournée vers l'extérieur qui fait appel aux capacités sensorimotrices des êtres humains et l'autre, tournée vers l'intérieur, qui permet de révéler les propriétés cognitives conceptuelles du cerveau.

9 Le langage doit être pensé prioritairement en lien avec les facultés de pensée et non avec la communication. Passons au chapitre suivant (11. « Universal Grammar »). La GU pousse dans le cerveau des enfants comme les dents poussent dans leur bouche. Ces développements sont génétiquement programmés dans l'espèce humaine. Contrairement aux dents, à la marche et à l'apparition des règles menstruelles, il semblerait toutefois que l'environnement joue un rôle non négligeable dans le développement du langage. Certes, reconnait Chomsky, mais l'environnement linguistique joue un rôle au niveau de l'interface externe du langage. L'interface interne est aussi génétiquement réglée que la marche. Il en résulte que toutes les langues sont capables d'exprimer toutes les pensées (langage et bipédie, même combat).

Le chapitre 12 traite d'acquisition du langage. Il vaudrait mieux parler de développement que d'apprentissage, nous dit Noam. Ce chapitre devient assez technique avec l'utilisation des termes liés au dernier avatar du modèle générativiste (Programme minimaliste, dernière proposition de Chomsky au milieu des années 1990). Le chapitre 13 est consacré aux aspects sémantiques du langage, qui ne sont pas au centre des réflexions chomskiennes, reconnait Salkie. Grosso modo, phonologie et sens sont étroitement liés : l'enfant qui entend table crée des liens dans son cerveau avec cable, sable, rable... Ainsi, lexique et phonologie croissent de pair. Cette approche 
décontextualisée du langage est la seule à supporter une approche scientifique selon Chomsky. On peut bien sûr s'intéresser à la pragmatique, «but [it] is unlikely to be amenable to scientific inquiry, in Chomsky's view » (p. 111).

11 Un chapitre sur l'évolution du langage présente l'hypothèse de Chomsky : il apparait suite à une mutation génétique. Cette mutation confère d'énormes avantages aux porteurs et porteuses du gène, pas seulement pour le langage mais également pour le développement des techniques et des arts. Elle est sélectionnée par l'évolution, et nous sommes tous et toutes les descendantes de ces premiers mutants.

12 La citation de Chomsky en exergue de l'avant dernier chapitre est un appel à la probité des intellectuels : "It is the responsability of intellectuals to speak the truth and to expose lies ", ce qui veut dire parler dans un langage clair et direct, sans adopter de position surplombante et narquoise, mais en montrant qu'il existe d'autres points de vue sur la réalité que celui des dominants qui tentent à faire accroire qu'il n'y a pas d'alternative à leur propre vision de la réalité.

13 Le $16^{\mathrm{e}}$ et dernier chapitre est consacré à l'héritage de Chomsky (mais il n'est pas encore mort !). Entre son influence en linguistique (finalement très faible) et en politique, il ne faut pas chercher de lien, si ce n'est la volonté de toujours interroger ce qui semble aller de soi, de se mettre en position minoritaire, finalement. Cet ultime chapitre contient des recommandations pratiques si vous voulez vous lancer dans l'activisme politique. Surtout : pensez par vous-même (donc pas forcément comme Chomsky).

14 La lecture de ce petit livre est plaisante, il s'adresse à un large public, l'auteur est plein d'humour, mais on a parfois l'impression qu'il s'agit d'un évangile de la vie et de la parole de Noam Chomsky.

\section{AUTEURS}

\section{MARINETTE MATTHEY}

Univ. Grenoble Alpes, LIDILEM, 38000 Grenoble, France 